\title{
NANOCHARACTERIZATION OF GALLIUM ANTIMONIDE SUBSTRATE SURFACE BY TEM/AFM
}

\author{
X. Li, ${ }^{*}$ L. Allen, ${ }^{* *}$, C. Santeufemio**, W. Goodhue***, and C. Sung ${ }^{*}$, \\ * Department of Chemical and Nuclear Engineering, Center for Advanced Materials, University of \\ Massachusetts, Lowell, MA 01854 \\ ** Epion, a JDS Uniphase Company, Epion Corporation, 37 Manning Rd, Billerica, MA. 01821 \\ *** Department of Physics, Photonics Center, University of Massachusetts, Lowell, MA 01854
}

Recently, antimonide-based compound semiconductor (ABCS) attracts more and more attention because of their opto-electronic applications in the mid-to-far-infrared region [1]. Efficient longwavelength semiconductor lasers operating at room temperature in the mid-infrared wavelength region $(2-5 \mu \mathrm{m})$ have great potential in chemical sensing applications, such as environmental monitoring and industrial process control. In addition, III-V antimonide compounds are proved to be promising candidates as barrier materials for many optical and electronic devices in infrared region [2]. To all the ABCS heterostructure compounds, $\mathrm{GaSb}$ is a good substrate material because it has the same lattice parameter with other ABCS, which is $0.61 \mathrm{~nm}$.

High performance GaSb-based devices rely on a high degree of perfection in both the bulk substrate and surface crystal quality. The interface between the substrate and the epitaxial active layer is critical to performance issues such as transistor current gain, ability to pinch off, and turn-on voltage. The ideal substrate would be a perfectly polished semi-insulating GaSb wafer. Gas Cluster Ion Beam (GCIB) technique is suitable to modify GaSb surface.

The goal of this research is to make a criterion for GaSb substrate characterization, and to improve the GaSb wafer to become "Epi-ready". That means treated wafers will be uniform, have no defect and have a specific oxidation layer, which can removed easily. Smooth wafer will have relatively low dislocation density such as 200 dislocations $/ \mathrm{cm}^{2}$. Atomic force microscopy (AFM) is used to characterize the substrate, which is smoothened by GCIB. Fig.1 and Fig. 2 show the effect of GCIB process. Many kinds of gas and different combinations of GCIB steps are performed. The best result is selected based upon the data obtained from AFM. In addition, X-ray photoelectron spectroscopy (XPS) is used to characterize the oxide layer upon GaSb substrate [3]. The growth interfaces are characterized by transmission electron microscopy (TEM) in conjunction with AFM and x-ray rocking curve method. TEM cross-section view can characterize the interface of growth wafer, especially in the groove and trench region. (Fig.3 and Fig. 4)

\section{References:}

1. J. Shin et al., Journal of Crystal Growth 179 (1997) 1

2. S. Li et al., Journal of Crystal Growth 223 (2001) 456

3. N. Kitamura, Materials Letters 29 (1996) 77 


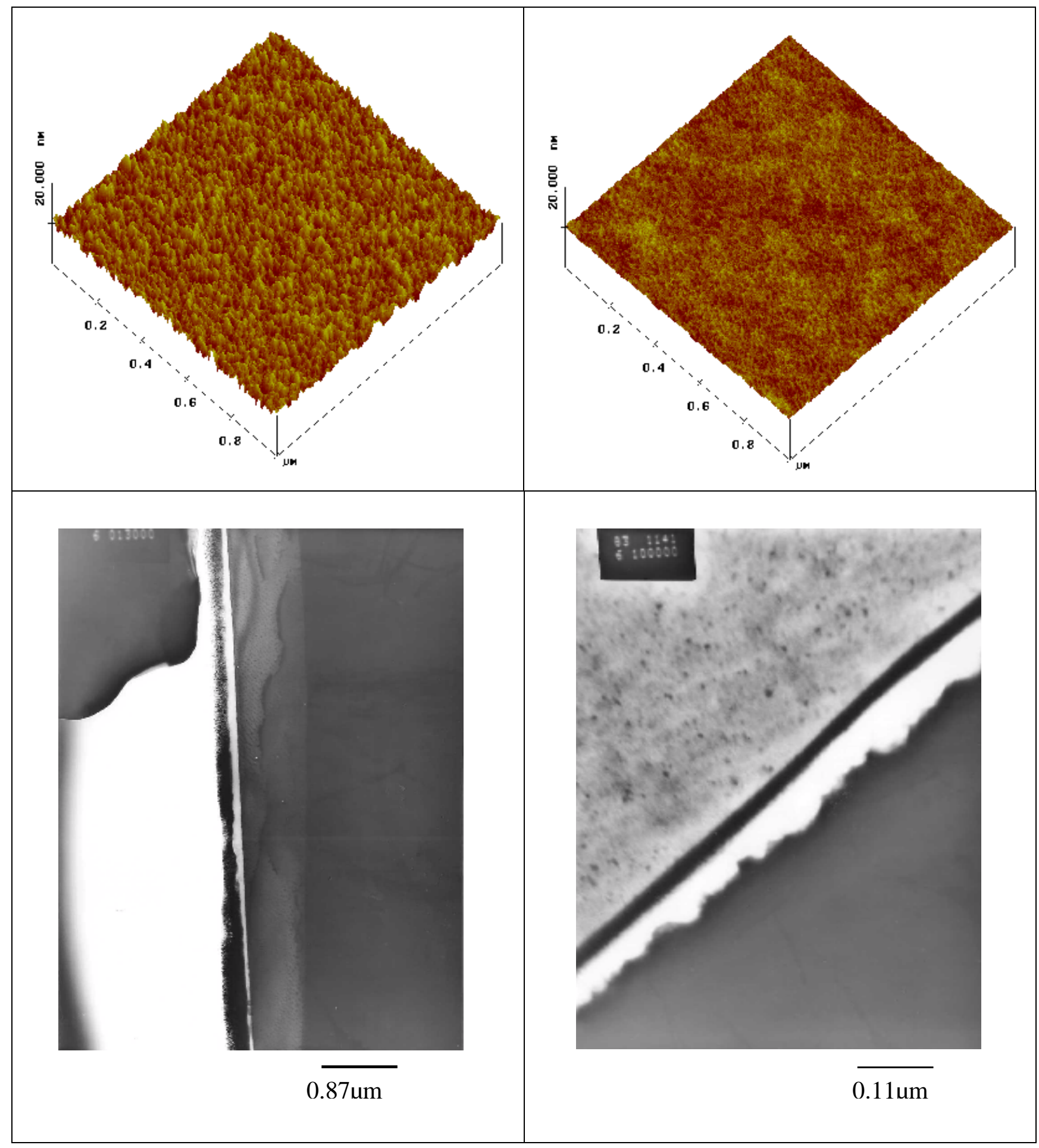

FIG. 1 AFM image showing surface morphology of original sample

FIG. 2 AFM image showing surface morphology of sample which is after the GCIB procedure FIG. 3 TEM cross-section of epi-substrate GaSb, showing smooth surface FIG. 4 TEM cross-section of epi-substrate GaSb, showing rough surface 